Article

\title{
Household, Personal and Environmental Correlates of Rural Elderly's Cycling Activity: Evidence from Zhongshan Metropolitan Area, China
}

\section{Yi Zhang ${ }^{1}$, Xiaoguang Yang ${ }^{2}$, Yuan $\mathrm{Li}^{3}$, Qixing Liu ${ }^{4}$ and Chaoyang $\mathrm{Li}^{1{ }^{1} * *}$}

1 School of Naval Architecture Ocean and Civil Engineering, Shanghai Jiao Tong University, 800 Dongchuan Road, 200240 Shanghai, China; E-Mail: darrenzhy@sjtu.edu.cn

2 Key Laboratory of Road and Traffic Engineering of Ministry of Education, Tongji University, 1239 Siping Road, 200092 Shanghai, China; E-Mail: yangxg@tongji.edu.cn

3 School of Architecture and Civil Engineering, Xiamen University, 422 South Siming Road, 361005 Xiamen, China; E-Mail: liyuan79@xmu.edu.cn

4 Rotterdam School of Management, Erasmus University, Burgemeester Oudlaan 50, 3062 PA Rotterdam, The Netherlands; E-Mail: qliu@mba15.rsm.nl

* Author to whom correspondence should be addressed; E-Mail: cyljjf@sjtu.edu.cn; Tel.: +86-21-6281-3386; Fax: +86-21-6281-3386.

Received: 18 April 2014; in revised form: 27 May 2014 / Accepted: 30 May 2014 /

Published: 5 June 2014

\begin{abstract}
Cycling is an important form of active transport and physical activity to provide substantial health benefits to the elderly. Among voluminous physical activity-related literature, few studies have investigated the correlates of active transport of the rural elderly in China. This study was the first attempt to investigate the impact of the household, personal, and environmental attributes on rural elderly's cycling activity with data collected in 102 rural neighborhoods of Zhongshan Metropolitan Area, China. The negative binomial regression models suggest that, all else being equal, living in a neighborhood with low proportion of elderly population (over 60), abundant bike lanes, and a compact urban form related to high density and mixed development, are associated with the increase of frequency and duration of the rural elderly's cycling trips. The models also detect that attitude towards cycling and household bicycle and motorized vehicle ownership are strongly related to cycling trips of the rural elderly in Zhongshan. The findings provide insights for transportation and public health agencies, practitioners, and researchers into the effective design of interventions from the prospective of attitudes, social and built environment on health promotion of the rural elderly in China.
\end{abstract}


Keywords: rural elderly; cycling activity; frequency and duration; negative binomial regression; social environment; built environment

\section{Introduction}

There is strong evidence that regular active transport provides substantial health benefits to the elderly [1]. Active transport, as a part of physical activity, is positively associated with prevention of chronic diseases, disability, and bone fractures among the elderly [2]. As an important form of active transport to promote in the Chinese elderly, cycling is accessible and easy to incorporate into daily life. To promote cycling among the elderly is a crucial component of efforts to improve their overall physical activity levels. Up to year 2012, the population of the elderly (aged 60 and over) in China was 194 million, accounting for $14.3 \%$ of the Chinese population [3]. Among China's elderly population, $60 \%$ live in the rural area. It is a challenge to promote active transport among the China's rural elderly as the motorization has been spread from the urban areas to the rural ones and the mode split of active transport is keeping shrinking. Since 2007, the National Health and Family Planning Commission of China (NHFPC) has launched an initiative of "Ten Thousand Steps a Day" for the Chinese elderly [4], aiming to promote all kinds of walking activities. However, no interventions with regard to the promotion of cycling activity have been implemented. As a popular travel mode in China's rural areas, the evidence about the correlates that may impact on elders' cycling activity is scarce, leading to the lack of practical implications for the planning of environmental interventions.

The transportation planning fields [5,6] and the public health field [7-9] have mutually contributed to the proliferation of research on the environmental correlates of active transport (especially walking) by the elderly in the western context [10]. The research findings from the two fields have facilitated our understanding on the connection between the environmental correlates and active transport by the elderly and provided important policy implications for the planning of interventions. To be specific, the environmental correlates in previous literature are defined as two categories, the social environment and the built environment. The social environment "includes the culture that the individual was educated or lives in, and the people and institutions with whom they interact" [11]. The built environment is defined as "the human-made space in which people live, work, and recreate on a day-to-day basis" [12] and "encompasses places and spaces created or modified by people including buildings, parks, and transportation systems" [13].

In a recent systematic review mainly focusing on the Western context, Van Cauwenberg et al. [9] concluded that the following built environment features would impact active transport of the elderly, albeit to varied degrees: (1) walkability, e.g., residential density, land use mix diversity, and street connectivity $[14,15]$; (2) access to services, e.g., access to public transportation and recreational facilities [16,17]; (3) walking facilities, e.g., sidewalks and walking trails [16]; (4) safety, e.g., presence of heavy traffic and neighborhood crime-related safety [14,16-19]; (5) aesthetics, e.g., greenery and scenery [18]; and (6) urbanization, e.g., the difference between urban and rural residents [15,20]. Similarly, Saelens et al. found that neighborhoods with higher density, greater connectivity, and more land use mix report higher rates of walking/cycling for utilitarian purposes than 
low-density, poorly connected, and single land use neighborhoods [6]. In terms of the effects of social environment, the role models and neighborhood social cohesion were found to related to walking time, and peer support would influence older adults' physical activity [21,22].

The environmental attributes employed in active transport related studies were typically derived [23] by: (1) surveying individuals' perceptions of the social or built environment [24,25]; (2) aggregating neighborhood measures from secondary data, such as Census or Traffic Analysis Zone [26-30]; (3) measuring these characteristics within a certain distance of the individuals' residences [31,32], e.g., by buffer radii (ranging from $100 \mathrm{~m}$ to $1 \mathrm{~km}$ ); or (4) quantifying the built environment attributes objectively at high resolution or used cluster analysis to identify different urban forms $[27,28,33]$. Over the past decade, the volume of literature on the influence of the environment on physical activity and health promotion among different age groups in the Western context has exploded.

Worth mentioning is that the majority of "environment-physical activity" studies were predominantly conducted in Western contexts and their findings are not necessarily translatable to the Chinese contexts [9]. In recent years, Chinese scholars began to examine the effects of various effects on the health level of different age groups [34]. However, rare studies have examined the environmental representations in the Chinese context, let alone the association between the environment attributes and cycling activity among the rural elderly, as the present paper does. Since the cycling activity of the rural elderly is an indispensable starting point to facilitate the understanding and design effective interventions on health promotion, the present paper will serve as an extended body of literature.

This study makes an important contribution to the literature. The correlates of the cycling activity of the rural elderly in Zhongshan, China, are investigated in an effort to better understand the impact of the household, personal and environmental attributes on such physical activity. To our knowledge, this is the first such study on the subject in the Chinese context. Firstly, the study generated four categories of attributes: household, personal, social environmental, and built environmental attributes. Then, negative binomial regression models were used to examine specifically how the frequency and duration of rural elderly's cycling trips are related to the social and built environment attributes, together with personal and household attributes. The cycling activity in the present study includes both transportation and recreational cycling. The elderly population in this study focus on the older adults aged 60 and over. The findings will provide insights for transportation and public health agencies, practitioners, and researchers into the effective design of interventions on health promotion of the rural elderly.

\section{Data and Methods}

\subsection{Study Area}

As stated in previous studies [26,29], the Zhongshan Metropolitan Area was chosen to examine the cycling behaviors of the rural elderly in the Chinese context. Zhongshan is a medium-sized prefecture-level city in Guangdong Province of southern China (Figure 1). In the three largest coastal urban agglomerations with the most competitive economies in China, there are about 20 medium-sized cities with similar urbanization and motorization level, as well as urban and rural transport characteristics to Zhongshan [35]. Thus, the research findings in Zhongshan might be typical and informative to the type of cities. In Zhongshan, the elderly make 2.55 trips per day in average, among 
which $18.0 \%$ were cycling trips. The average frequency and duration of cycling trips of the elderly is 0.46 times and 5.90 minutes [36].

Figure 1. Study area.

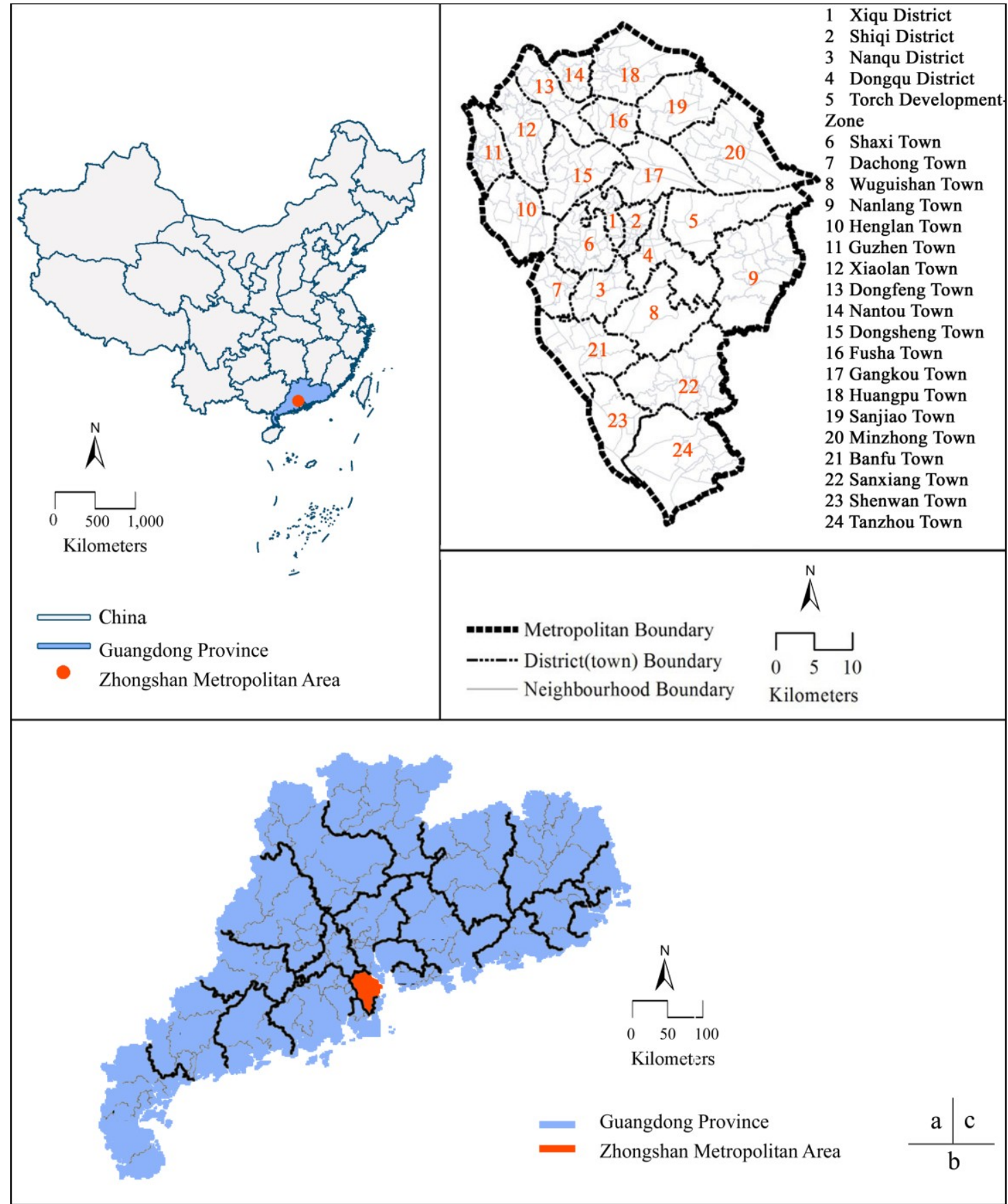

Note: a shows the location of Guangdong Province and Zhongshan Metropolitan Area in China; b shows the location of Zhongshan Metropolitan Area in Guangdong Province; and c shows the metropolitan boundary, district (town) boundary, neighborhood boundary, and the location of all 24 districts (towns) in Zhongshan Metropolitan Area.

\subsection{Data Collection}

The cross-sectional cycling activity data were derived from Zhongshan Household Travel Survey (ZHTS) in 2010 [36]. Selected by stratified random sampling covering the whole Zhongshan Metropolitan 
Area, the sample size of the rural elderly over 60 was 1572 (excluding invalid data) from 102 rural neighborhoods, with a sample rate of $2.0 \%$. The ZHTS 2010 provided the self-reported one-day cycling activity, e.g., frequency, duration, purpose of cycling trips, together with the personal and household data of the elderly.

The following data for the characterization of social and built environment attributes come from Zhongshan Municipal Bureau of Urban Planning [26,29]: (1) traffic analysis zones' boundaries - proxy for neighborhood boundaries; (2) land use in 2010 with five major types of land use (residential land, commercial and service facilities, industrial and manufacturing, green space, and other types of land uses); (3) population, dwelling units and employment in 2010; (4) road networks; (5) bus stops; (6) political boundaries, such as city and zone boundaries; and (7) traffic analysis zone level of socio-demographic data. All the data were then integrated into ArcGIS for further analysis.

\subsection{Characterization of Social Environment Attributes}

The social and built environment attributes were characterized on the basis of neighborhoods, which are defined to be spatially equivalent to traffic analysis zones. As designed to be homogeneous with respect to socio-demographic characteristics and living conditions [37], traffic analysis zones share boundaries with administrative divisions in most cases. According to the administrative divisions of Zhongshan Metropolitan Area, a total of 102 rural traffic analysis zones (neighborhoods) were selected in this study, although three neighborhoods out of the 102 are highly populated with similar population density (over 8000 persons $/ \mathrm{km}^{2}$ ) to the average density of urban neighborhoods in Zhongshan. With respect to previous literature, we defined three attributes to demonstrate the social environment of the neighborhood where the rural elderly live, i.e., the proportions of employed population, the proportions of elderly population, and the average household income.

\subsection{Characterization of Built Environment Attributes}

In Ewing and Cervero's research [33], the built environment variables that influenced travel behavior were named with words beginning with D as "five Ds" from five aspects: density, design, distance to transit, destination accessibility, and diversity. Each D variable contains a number of attributes that are commonly used in travel behavior-built environment research (Table 1). In this study, considering the best available data, we identified five built environment attributes in response to the five Ds: population density (density), bike lane density (design), bus service coverage rate (distance to transit), distance to the central business district (destination accessibility), and land-use diversity (diversity). The term of bike lane in this study referred to two major types of bike facilities in the rural areas of Zhongshan: (1) the separated bike lanes that are physically separated from car traffic by a curb, or fence, or trees, and (2) the bike lanes marked by a painted white line on a road meant for cars. Both of the two types are at least $1.5 \mathrm{~m}$ wide (for one direction) according to the official design criteria in China. In terms of the built environment features that would also impact the active transport of the elderly [9], e.g., safety, aesthetics, or urbanization, we did not employ them in this study due to the limited available data. 
Table 1. The meaning and commonly used attributes of five Ds built environment variables.

\begin{tabular}{lll}
\hline Five Variables & Meaning & Commonly used attributes \\
\hline Density & $\begin{array}{l}\text { The variable of interest } \\
\text { per unit of area }\end{array}$ & $\begin{array}{l}\text { Population density, dwelling units density, } \\
\text { employment density }\end{array}$ \\
\hline Design & $\begin{array}{l}\text { Street network characteristics } \\
\text { within an area }\end{array}$ & $\begin{array}{l}\text { Average block size, proportion of four-way } \\
\text { intersections, number of intersections per square } \\
\text { mile, bike lane density, average building setbacks, } \\
\text { average street widths, numbers of pedestrian } \\
\text { crossings, street trees }\end{array}$ \\
\hline Diversity & $\begin{array}{l}\text { The number of different } \\
\text { land uses in a given area } \\
\text { and the degree to which } \\
\text { they are represented }\end{array}$ & $\begin{array}{l}\text { Entropy measures of diversity, jobs-to-housing } \\
\text { ratios, jobs-to-population ratios }\end{array}$ \\
\hline Distance to transit & $\begin{array}{l}\text { The level of transit service at } \\
\text { the residences or workplaces }\end{array}$ & $\begin{array}{l}\text { Distance from the residences or workplaces to the } \\
\text { nearest rail station or bus stop, transit route density, } \\
\text { distance between transit stops, number of stations } \\
\text { per unit area, bus service coverage rate }\end{array}$ \\
\hline $\begin{array}{l}\text { Destination } \\
\text { accessibility }\end{array}$ & $\begin{array}{l}\text { Ease of access to } \\
\text { trip attractions }\end{array}$ & $\begin{array}{l}\text { Distance to the central business district, number } \\
\text { of jobs or other attractions reachable within a given } \\
\text { travel time, distance from home to the closest store }\end{array}$ \\
\hline
\end{tabular}

The population density, bike lane density, and distance to the central business district are self-explanatory. The bus service coverage rate and land-use diversity were calculated with respect to the context of Zhongshan or China, as applicable. The bus service coverage rate, calculated by the ratio of bus service coverage to total area of neighborhood, reflects the level of transit services. In Chinese cities, the transit service area is defined as a 300-meter radius of each transit station [38]. The land-use diversity represents the degree to which different land uses in a neighborhood are mixed. We calculated the land-use diversity by the Entropy Index (EI) [39], wherein 0 indicates single-use environments and 1 stands for the equalization of different land uses in area coverage. EI is defined by:

$$
E I=\sum_{i=1}^{n} P_{i} \log \left(1 / P_{i}\right)
$$

where $n=$ number of unique land uses, $n \geq 1 ; P_{i}=$ percentage of land use $i$ 's coverage over total land use coverage. In China, the officially recommended proportion of residential land, industrial manufacturing land, commercial facilities land, green space land and other land is close to 2:2:1:1:1 [40], which is applied in the land use planning practice in Chinese cities including Zhongshan. This proportion generates an entropy index of 0.67 . Thus each of the original entropy index of a traffic analysis zone in this study is transformed in a criteria that 0.67 is the standard 1 , and all other index are ranged between 0 and 1 based on the standard 1 [26].

\subsection{Model Specification}

Because the rural elderly's trips are non-negative count dependent variables, we tested the data to choose the proper models. Due to the characteristics of the dependent variables (Table 1), we preferred 
negative binomial regression to poisson regression. The Vuong model selection test strongly favoring a standard negative binomial regression approach than a zero-inflated one to analyzing the data.

This study chose a negative binomial regression model to analyze the relationship between the households, personal and environmental attributes and the rural elderly's cycling activity. To calibrate coefficient of negative binomial regression model with Stata 12.0, we rewrote the negative binomial regression model with natural log transform. We employed the same independent variable sets in the frequency and duration of rural elderly's cycling trips. The basic negative binomial regression model specifications were expressed as follow:

$$
\begin{aligned}
& N r_{\text {frequency }}\left(N r_{\text {duration }}\right)=\beta_{0}{ }^{\prime}+\beta_{1}{ }^{\prime} * \text { HHSIZE_ } 1+\beta_{2}{ }^{\prime} * \text { HHSIZE } 22+\beta_{3}{ }^{\prime} * \text { HIGHINC }+\beta_{4}{ }^{\prime} \\
& * \text { MEDINC }+\beta_{5}{ }^{\prime} * \text { N_EMPLOYED }+\beta_{6}{ }^{\prime} * \text { BIKES }+\beta_{7}{ }^{\prime} * \text { EBIKES }+\beta_{8}{ }^{\prime} * \text { MOTORS }+ \\
& \beta_{9}{ }^{\prime} * \mathrm{CARS}+\beta_{10}{ }^{\prime} * \mathrm{GENDER}+\beta_{11}{ }^{\prime} * \mathrm{AGE}+\beta_{12}{ }^{\prime} * \mathrm{PROBIKE}+\beta_{13}{ }^{\prime} * \mathrm{PROWALK}+ \\
& \beta_{14} \text { * PROEBIKE }
\end{aligned}
$$

where $N r_{\text {frequency }}$ and $N r_{\text {duration }}$ are frequency (times/day) and duration (minutes/day) of cycling trips; HHSIZE_1 and HHSIZE_2 are dummies for the household size of one and two (with a reference category of more than two); HIGHINC and MEDINC_M are dummies for the household total annual income ranges of above 60,000 Chinese Yuan (Renminbi) (RMB, 6.2 Renminbi $\approx 1$ US Dollar) and 20,000-60,000 RMB (with a reference category of 0-20000 RMB); BIKES, EBIKES, MOTORS, and CARS represent the number of bicycles, electric bikes, motorcycles and private cars in a household, respectively; GENDER denotes whether the respondents is male or female; AGE means the respondent's age in years; PROBIKE, PROWALK, or PROEBIKE demonstrates whether the respondents favor bicycle, walking or e-bike over other travel modes in daily travel.

Along with the basic model presented above, regression of the dependent variables proceeded in an expanded model. The expanded model adds four social environment and five built environment attributes as independent variables, where R_EMPLOYED, R_ELDERLY, R_HIGHINC, and R_MEDINC denotes the ratio of the employed population, elderly population, high-income households and medium-income households in the neighborhood, respectively; POP, BIKELANE, BUSSERV, CBDDIST, and MIXTURE demonstrate the population density, bike lane density, bus service coverage rate, distance to the central business district, and land-use mixture of the neighborhood where the respondents lives.

\section{Results}

\subsection{Descriptive Statistics}

Descriptive statistics provide a general view of the dependent and independent variables (Table 2). Nearly one-quarter of the respondents live alone and over a third live with a partner. $88 \%$ of the respondents live in the medium-to-low-income households. The household ownership of bicycle averaged 0.60, a little lower than that of motorcycle. Sixty-three percent of the respondents were male because of the low rate of response of female elderly. One-sixth of the total respondents favored bicycle over other non-motorized modes. The average age of the respondents was 67.17. 
The standard deviation values of the built environment attributes were close to their mean values (all but MIXTURE, land-use mixture), implying the substantial variations of built environment features among rural neighborhoods in Zhongshan (Table 2).

Table 2. Descriptive statistics for dependent and independent variables in the 102 rural neighborhoods in Zhongshan Metropolitan Area in 2009 (sample size $=1572$ elderly).

\begin{tabular}{|c|c|c|c|c|c|}
\hline Variable & Description & Mean & S. D. & Min. & Max. \\
\hline \multicolumn{6}{|c|}{ Dependent variables } \\
\hline Frequency & Frequency of rural elderly's cycling trips, times per day, count & 0.46 & 1.00 & 0 & 6 \\
\hline Duration & Duration of rural elderly's cycling trips, minutes per day, count & 5.90 & 14.68 & 0 & 120 \\
\hline \multicolumn{6}{|c|}{ Household attributes (Independent variables) } \\
\hline HHSIZE_1 & Household size is one person, binary, $1=$ yes & 0.23 & 0.42 & 0 & 1 \\
\hline HHSIZE_2 & Household size is two persons, binary, $1=$ yes & 0.35 & 0.48 & 0 & 1 \\
\hline HHSIZE $>2$ & Household size is three or more persons, binary, $1=$ yes & 0.43 & 0.49 & 1 & 1 \\
\hline HIGHINC & High household income $(>60000 \mathrm{RMB} /$ year $)$, binary, $1=$ yes & 0.12 & 0.32 & 0 & 1 \\
\hline MEDINC & $\begin{array}{l}\text { Medium household income (20000-60000 RMB/year), } \\
\text { binary, } 1=\text { yes }\end{array}$ & 0.41 & 0.49 & 0 & 1 \\
\hline LOWINC & Low household income $(<20000 \mathrm{RMB} /$ year $)$, binary, $1=$ yes & 0.48 & 0.50 & 0 & 1 \\
\hline EMPLOYED & Number of persons employed in a household, count & 1.22 & 1.13 & 0 & 5 \\
\hline BIKES & Number of bikes in a household, count & 0.60 & 0.69 & 0 & 5 \\
\hline E-BIKES & Number of electric bikes in a household, count & 0.24 & 0.47 & 0 & 3 \\
\hline MOTORS & Number of motorcycles in a household, count & 0.70 & 0.84 & 0 & 5 \\
\hline CARS & Number of private cars in a household, count & 0.13 & 0.41 & 0 & 4 \\
\hline \multicolumn{6}{|c|}{ Personal demographic and attitudinal attributes (Independent variables) } \\
\hline GENDER & $1=$ Male, $0=$ Female, binary & 0.63 & 0.48 & 0 & 1 \\
\hline AGE & Age of the respondent in years, count & 67.17 & 6.77 & 60 & 95 \\
\hline PROBIKE & $\begin{array}{l}\text { The respondent favors bicycle over other non-motorized modes, } \\
\text { binary, } 1=\text { yes }\end{array}$ & 0.16 & 0.37 & 0 & 1 \\
\hline PROWALK & $\begin{array}{l}\text { The respondent favors walking over other non-motorized modes, } \\
\text { binary, } 1=\text { yes }\end{array}$ & 0.23 & 0.42 & 0 & 1 \\
\hline PROEBIKE & $\begin{array}{l}\text { The respondent favors e-bike over other non-motorized modes, } \\
\text { binary, } 1=\text { yes }\end{array}$ & 0.07 & 0.25 & 0 & 1 \\
\hline \multicolumn{6}{|c|}{ Social environment attributes (Independent variables) } \\
\hline P_EMPLOYED & $\begin{array}{l}\text { Proportions of employed population in the neighborhood, } \\
\text { continuous }\end{array}$ & 0.62 & 0.08 & 0.44 & 0.81 \\
\hline P_ELDERLY & $\begin{array}{l}\text { Proportions of elderly population in the neighborhood, } \\
\text { continuous }\end{array}$ & 0.13 & 0.06 & 0.05 & 0.28 \\
\hline P_HIGHINC & $\begin{array}{l}\text { Proportions of high-income household in the neighborhood, } \\
\text { continuous }\end{array}$ & 0.08 & 0.27 & 0 & 1 \\
\hline P_MEDINC & $\begin{array}{l}\text { Proportions of medium-income household in the neighborhood, } \\
\text { continuous }\end{array}$ & 0.67 & 0.47 & 0 & 1 \\
\hline P_LOWINC & $\begin{array}{l}\text { Proportions of low-income household in the neighborhood, } \\
\text { continuous }\end{array}$ & 0.25 & 0.43 & 0 & 1 \\
\hline
\end{tabular}


Table 2. Cont.

\begin{tabular}{llrrrr}
\hline Variable & Description & Mean & S. D. & Min. & Max. \\
\hline & \multicolumn{1}{c}{ Built environment attributes (Independent variables) } & & & & \\
\hline BIKELANE & Bike lane density, $\mathrm{km} / \mathrm{km}^{2}$, continuous & 2.58 & 2.09 & 0.25 & 9.58 \\
\hline POPDEN & Population density, 1000 persons $/ \mathrm{km}^{2}$, continuous & 2.31 & 2.06 & 0.16 & 13.00 \\
\hline MIXTURE & Land-use mixture & 0.67 & 0.17 & 0.07 & 0.99 \\
\hline BUSSERV & Bus stop density, number of bus stops per $\mathrm{km}^{2}$, continuous & 0.28 & 0.21 & 0.00 & 0.96 \\
\hline \multirow{2}{*}{ CBDDIST } & $\begin{array}{l}\text { Euclidean distance from the centroid of the neighborhood to } \\
\text { the central business district, in km, continuous }\end{array}$ & 2.01 & 1.03 & 0.04 & 4.29 \\
\hline
\end{tabular}

Note: S. D. = Standard Deviation; Min. = minimum; Max. = maximum.

\subsection{Negative Binomial Regression Analysis of Frequency and Duration of Rural Elderly's Cycling Trips}

The results of negative binomial regressions indicated how different demographic, attitudinal and environmental attributes were related to the cycling frequency and duration of the rural elderly (Table 3). It is worth mentioning that as the results were derived from cross-sectional data, it is hard to know the cause and effect relationship. For example, in previous studies, more bike lanes were highly correlated to more cycling $[41,42]$. However, in this study, even if we had similar findings, it is arbitrary for us to simply conclude that bike lanes would promote more cycling. There is still a possibility that bike lanes were built afterwards to accommodate pre-existing high numbers of cyclists.

Table 3. Negative binomial regressions of frequency and duration of cycling trips of the rural elderly in Zhongshan.

\begin{tabular}{|c|c|c|c|c|}
\hline \multirow{3}{*}{ Variable } & \multicolumn{4}{|c|}{ Coefficients by Dependent Variables and Model Type } \\
\hline & \multicolumn{2}{|c|}{ Frequency of cycling (times/day) } & \multicolumn{2}{|c|}{ Duration of cycling (min./day) } \\
\hline & Basic & Expanded & Basic & Expanded \\
\hline \multicolumn{5}{|c|}{ Household attributes (HHSIZE $>2$ and LOWINC are reference categories) } \\
\hline HHSIZE_1 & 0.282 & 0.229 & 0.603 & 0.553 \\
\hline HHSIZE_2 & $0.297 * * *$ & 0.272 & $0.605 * * *$ & $0.512 * * *$ \\
\hline HIGHINC & 0.178 & 0.018 & 0.385 & 0.499 \\
\hline MEDINC & 0.153 & 0.033 & 0.143 & 0.291 \\
\hline N_EMPLOYED & $0.154 *$ & $0.133 * *$ & 0.092 & 0.038 \\
\hline BIKES & $0.937 *$ & $0.921 *$ & $1.733 *$ & $1.993 *$ \\
\hline EBIKES & $-0.283 * *$ & $-0.264 * * *$ & $-0.479 * * *$ & $-0.470 * * *$ \\
\hline MOTORS & $-0.211 * *$ & $-0.169 * *$ & -0.087 & -0.130 \\
\hline CARS & $-0.328 * *$ & $-0.342 * *$ & -0.595 & $-0.786 * * *$ \\
\hline \multicolumn{5}{|c|}{ Personal attributes } \\
\hline MALE & $0.395 *$ & $0.349 *$ & $0.402 * *$ & $0.357 * * *$ \\
\hline AGE & $-0.030 *$ & $-0.030 *$ & $-0.055 *$ & $-0.039 * *$ \\
\hline PROBIKE & $1.359 *$ & $1.454 *$ & $1.889 *$ & $1.932 *$ \\
\hline PROWALK & $-0.828 *$ & $-0.766^{*}$ & $-0.734 *$ & $-0.813 *$ \\
\hline PROEBIKE & $-2.361 *$ & $-2.358 *$ & $-0.385 *$ & -0.003 \\
\hline
\end{tabular}


Table 3. Cont.

\begin{tabular}{|c|c|c|c|c|}
\hline \multirow{3}{*}{ Variable } & \multicolumn{4}{|c|}{ Coefficients by Dependent Variables and Model Type } \\
\hline & \multicolumn{2}{|c|}{ Frequency of cycling (times/day) } & \multicolumn{2}{|c|}{ Duration of cycling (min./day) } \\
\hline & Basic & Expanded & Basic & Expanded \\
\hline \multicolumn{5}{|c|}{ Social environment ( $\mathrm{R} \_$LOWINC is a reference category) } \\
\hline P_EMPLOYED & & $-1.448 * * *$ & & 0.362 \\
\hline P_ELDERLY & & $-4.379 *$ & & $-5.183 * *$ \\
\hline P_HIGHINC & & 0.208 & & 0.021 \\
\hline P MEDINC & & $0.313 * *$ & & -0.160 \\
\hline \multicolumn{5}{|c|}{ Built environment } \\
\hline BIKELANE & & $0.052 * *$ & & $0.134 * *$ \\
\hline POPDEN & & $0.074 * *$ & & $0.131 * * *$ \\
\hline MIXTURE & & $0.536 * * *$ & & $1.416 * *$ \\
\hline BUSSERV & & $-0.616 *$ & & $-0.792 * * *$ \\
\hline CBDDIST & & $-0.132 *$ & & -0.141 \\
\hline cons & -0.544 & 0.834 & 2.507 & 1.383 \\
\hline \multicolumn{5}{|c|}{ Summary statistics } \\
\hline Number of obs & 1572 & & & \\
\hline LR chi $^{2}$ & 450.74 & 481.86 & 184.42 & 200.91 \\
\hline Prob $>$ chi $^{2}$ & 0.0000 & 0.0000 & 0.0000 & 0.0000 \\
\hline Pseudo-R ${ }^{2}$ & 0.1704 & 0.1822 & 0.0415 & 0.0452 \\
\hline Log likelihood & -1096.8063 & -1081.1096 & -2128.8315 & -2120.584 \\
\hline
\end{tabular}

At the household level, household size, number of employment and vehicle ownership showed significant associations. Living in a household of two persons or with more persons employed is related to more and longer cycling trips. As expected, having an additional bike in a household was related to a $155.30 \%(=\exp (0.937)-1)$ or $466.04 \%$ increase in frequency or duration of cycling trips. Having an additional e-bike, motorcycle, or car was associated with $19.02 \%$ to $27.99 \%$ decrease of frequency of cycling trips.

At the personal level, all the demographic and attitudinal attributes showed significance at 95\% confidence. Being female or older in age was related to less cycle trips. The rural elderly's attitudes towards different non-motorized modes may play a remarkable role in their cycling trips. Those who preferred cycling to other modes would make $289.42 \%$ or $561.14 \%$ more cycling trips in terms of frequency or duration than the counterpart. Likewise the rural elderly who favored walking or e-bike over bicycle would make significantly less cycling trips.

At the social environment level, three attributes out of four showed significant associations with the frequency while only one with the duration. Living in a rural neighborhood with higher ratio of elderly population, the elderly would make $27.56 \%$ (frequency) or $27.75 \%$ (duration) fewer cycling trips at most. The frequency of cycling trips is also significantly related to the ratio of the employed population and medium-income households. 
At the built environment level, nearly all attributes were statistically significant at $90 \%$ confidence (except for the CBDDIST in the duration of the cycling trips). The rural elderly who live in the most-populated environment would make $99.02 \%$ (frequency) or $179.87 \%$ (duration) more cycling trips than in the least-populated environment. Similarly, living in the environment with the highest land-use mixture, the rural elderly would at most make $65.27 \%$ (frequency) or $286.96 \%$ (duration) more cycling trips. Denser bike lanes were related to more cycling activity among the rural elderly in Zhongshan. In the environment with the densest bike lanes, the rural elderly would make $49.99 \%$ (frequency) or $133.88 \%$ (duration) more cycling trips than in the environment with the sparsest. Convenient bus service and was related to greater distance to the central business district were associated with fewer cycling trips.

The directions of the effects for the household and personal attributes persisted across all models and the coefficients showed slight to moderate variation. The LR chi $^{2}$ and Log likelihood of each model represents the overall goodness of fit. The changes of Pseudo- $\mathrm{R}^{2}, \mathrm{LR} \mathrm{chi}^{2}$ and Log likelihood (Table 3) in expanded models implied that the social and built environment contributed to strengthening the explanatory power and enhanced the predictability of the models.

\section{Discussion and Policy Implications}

This study adds to the existing literature by exploring the relationship between household, personal and environmental attributes and rural elderly's cycling activity in the Chinese context with data collected in 102 rural neighborhoods of Zhongshan Metropolitan Area. The study generated household, personal and environmental attributes, including demographics, attitudes towards different travel modes, social environment, and built environment. Then, negative binomial regression models were used to examine specifically how the frequency and duration of rural elderly's cycling trips are related to the built environment attributes, together with personal and household attributes.

Household and personal attributes related to the living arrangement, the availability of vehicles, gender and age, and the attitude towards travel modes are highly associated to the rural elderly's cycling frequency and duration. To be specific, being male or younger-old, favoring cycling than other modes in daily travel, living with a partner, owing more bikes, or owning less cars (or motorcycles), is related to the increasing frequency and duration of rural elderly's cycling activity. The findings on the correlation of attitude imply the potential effects of the dissemination of health life style involving cycling activity among the rural elderly. The negative associations of motorized vehicles on cycling demonstrate the potential effects of policies that discourage vehicle ownership.

The correlations of the social and built environment attributes on the cycling activity of the rural elderly in Zhongshan yield some interesting findings. The social environment attribute relating to the degree of aging of the neighborhood was negatively associated with the cycling activity. Specifically, in a neighborhood with less elderly population, the elderly would have more cycling trips in terms of both frequency and duration. The reasons to this results remains unclear from the data we already had. According to some previous studies, it may be related to the associations of social norms with physical activity [43]. Another possible reason may be that the fit and healthy elderly choose to live in areas with younger people. Although the underlying reasons need further investigation in following studies, the result may imply that developing neighborhoods with relatively balanced age structure could be a 
potential intervention to promote cycling activity among the rural elderly. That may also challenge the idea of forming elderly community as the elderly are less likely to have cycling trips in such a social environment. The built environment attributes featuring cycling facility, land-use density, and diversity showed significant correlation to the frequency and duration of rural elderly's cycling trips, albeit to varied degrees. Abundant and accessible bike lanes were positively related to more cycling among the elderly in Zhongshan, consistent with previous findings [41,42]. In populated and mixed-developed neighborhood, the rural elderly tend to cycle more in terms of the frequency and duration. That may because the compact urban form, closely related to high population density and land use mixture, increases the possibility of having short-to-medium distance trips instead of long distance ones. The average trip distance of a single walking and cycling trip of rural elderly in Zhongshan are around 0.8 and $2.5 \mathrm{~km}$, respectively. Therefore, cycling is a competitive choice for medium-distance trips ranging from 2 to $3 \mathrm{~km}$, which is very likely to go beyond a single walking trip's reach.

To promote the cycling activity among rural elderly, it would be a great challenge to incorporate health interventions into the policy-making and practice of land-use and transportation planning and other public policies in China. From the discussions above, we could find out that interventions involving the personal attitudes, the social environment, and the built environment may be potentially effective in the Chinese context to increase the frequency and duration of cycling trips among the rural elderly. The findings in the paper provide insights for transportation and public health agencies, practitioners, and researchers into four possible interventions as below.

- Disseminating healthy life style involving cycling activity. As it is hard to change attitudes among the elderly, we recommended diversified initiatives (public lectures, cycling campaigns, specialized websites, etc.) that have been proven to be successful in the Ten Thousand Steps a Day program [44]. We even recommend incorporating the promotion of cycling activity into the Ten Thousand Steps a Day program.

- Developing neighborhoods with relatively balanced age structure and avoiding overly aggregation of the elderly population.

- Discouraging vehicle ownership. The findings in this study indicated that more vehicle ownership is related to less cycling, consistent with a previous study in the Chinese context [41]. It is recommended to encourage cycling through policies discourage vehicle ownership.

- Maintaining a compact urban form related to high density and mixed development.

\section{Strengths and Limitations}

This study has a number of strengths and limitations. In terms of the strengths, firstly, the study focused on the rural elderly population and provided informative policy implications for the aging society. Secondly, the study revealed the household, personal and environmental correlates of rural elderly's cycling activity in a developing country with rapid urbanization and motorization, which might further promote the comparative research between different contexts. In terms of the limitations, firstly, the dependent outcome, cycling activity, is based on self-reports, and therefore, may be subject to self-report bias that does not capture all domains of this activity. However, self-reports in physical activity are common in the field and remain the primary source for assessing cycling activity in large-scale studies like this. Secondly, cross sectional data were used in this study. The full evaluation of causal 
inferences about household, personal and environmental effects on cycling activity will require longitudinal and multilevel analyses of physical activity over time.

\section{Conclusions}

This study makes an important contribution to the literature. The correlates of the cycling activity of the rural elderly in Zhongshan, China, are investigated in an effort to better understand the possible impact of the household, personal and environmental attributes on such physical activity. To our knowledge, this is the first such study on the subject in the Chinese context. Firstly, the study generated four categories of attributes as independent variables: household, personal, social environmental, and built environmental attributes. Then, negative binomial regression models were used to examine specifically how the frequency and duration of rural elderly's cycling trips are related to the social and built environment attributes, together with personal and household attributes.

The research findings suggest that positive attitude towards cycling and the availability of household bicycles are strongly related to the more cycling trips of the rural elderly in Zhongshan. The findings on the correlation of attitude imply the potential effects of the dissemination of health life style involving cycling activity among the rural elderly. With respect to the social and built environment, all else being equal, living in a neighborhood with low proportion of elderly population, abundant bike lanes, and a compact urban form related to high density and mixed development, are associated with the increase of rural elderly's cycling activities.

The findings provide insights for transportation and public health agencies, practitioners, and researchers into the effective design of potential interventions from the prospective of attitudes, social and built environment on health promotion of the rural elderly in China. Among the possible interventions, we suggested to (1) disseminate healthy life style involving cycling activity; (2) develop neighborhoods with relatively balanced age structure; (3) discourage car ownership; and (4) maintain a compact land-use development.

\section{Acknowledgment}

The authors acknowledge the financial support of Project 12\&ZD203 by National Social Science Foundation of China, Project 51308336 by National Natural Science Foundation of China, Project 2013M541522 by China Postdoctoral Science Foundation, and Project 12TS15 by Social Science Foundation of Shanghai Jiao Tong University.

\section{Author Contributions}

In this paper, Yi Zhang designed the research programs, participated in the data collection, characterized the household and personal attributes, analyzed the results, and completed the writing of the introduction, results and discussion; Xiaoguang Yang committed to the data collection, the calculation of social environment attributes, the choice of proper modeling methods, and the writing of corresponding parts; Yuan Li carried out the characterization of the built environment measures and completed the writing work of corresponding parts; Qixing Liu carried out the data process and 
completed the writing work of corresponding parts; Chaoyang Li conceived and developed the research ideas and led the research in general.

\section{Conflicts of Interest}

The authors declare no conflict of interest.

\section{References and Notes}

1. Nelson, M.E.; Rejeski, W.J.; Blair, S.N.; Duncan, P.W.; Judge, J.O.; King, A.C.; Macera, C.A.; Castaneda-Sceppa, C. Physical activity and public health in older adults: Recommendation from the American College of Sports Medicine and the American Heart Association. Med. Sci. Sport. Exer. 2007, 39, 1435-1445.

2. Keysor, J.J. Does late-life physical activity or exercise prevent or minimize disablement?: A critical review of the scientific evidence. Am. J. Prev. Med. 2003, 25, 129-136.

3. Wu, Y. China Report of the Development on Aging Cause; Social Sciences Acadamic Press: Beijing, China, 2013. (In Chinese)

4. National Health and Family Planning Commission of China. Promotiona of Healthy Living Style in China; National Health and Family Planning Commission of China (NHFPC): Beijing, China, 2007. (In Chinese)

5. Handy, S.L.; Boarnet, M.G.; Ewing, R.; Killingsworth, R.E. How the built environment affects physical activity: Views from urban planning. Am. J. Prev. Med. 2002, 23, 64-73.

6. Saelens, B.E.; Sallis, J.F.; Black, J.B.; Chen, D. Neighborhood-based differences in physical activity: An environment scale evaluation. Am. J. Public Health 2003, 93, 1552-1558.

7. Brownson, R.C.; Hoehner, C.M.; Day, K.; Forsyth, A.; Sallis, J.F. Measuring the Built Environment for Physical Activity State of the Science. Am. J. Prev. Med. 2009, 36, S99-S123.

8. McCormack, G.R.; Shiell, A. In search of causality: A systematic review of the relationship between the built environment and physical activity among adults. Int. J. Behav. Nutr. Phys. Activity 2011, 8, 125-135.

9. Van Cauwenberg, J.; de Bourdeaudhuij, I.; de Meester, F.; van Dyck, D.; Salmon, J.; Clarys, P.; Deforche, B. Relationship between the physical environment and physical activity in older adults: A systematic review. Health Place 2011, 17, 458-469.

10. Saelens, B.E.; Handy, S.L. Built environment correlates of walking: A review. Med. Sci. Sport. Exer. 2008, 40, S550-S566.

11. Barnett, E.; Casper, M. A definition of "social environment". Am. J. Public Health 2001, 91, 465.

12. Roof, K.; Oleru, N. Public health: Seattle and King County's push for the built environment. J. Environ. Health 2008, 71, 24-27.

13. Srinivasan, S.; O'Fallon, L.R.; Dearry, A. Creating healthy communities, healthy homes, healthy people: Initiating a research agenda on the built environment and public health. Am. J. Public Health 2003, 93, 1446-1450.

14. Li, F.; Fisher, K.J.; Brownson, R.C.; Bosworth, M. Multilevel modelling of built environment characteristics related to neighbourhood walking activity in older adults. J. Epidemiol. Commun. Health 2005, 59, 558-564. 
15. Kemperman, A.; Timmerman, H. Influences of built environment on walking and cycling by latent segments of aging population. Transport. Res. Rec. 2009, 2134, 1-9.

16. Nagel, C.L.; Carlson, N.E.; Bosworth, M.; Michael, Y.L. The relation between neighborhood built environment and walking activity among older adults. Am. J. Epidemiol. 2008, 168, 461-468.

17. Sugiyama, T.; Thompson, C.W. Older people's health, outdoor activity and supportiveness of neighbourhood environments. Landsc. Urban Plan. 2007, 83, 168-175.

18. Sugiyama, T.; Thompson, C.W.; Alves, S. Associations between neighborhood open space attributes and quality of life for older people in Britain. Environ. Behav. 2009, 41, 3-21.

19. De Leon, C.F.M.; Cagney, K.A.; Bienias, J.L.; Barnes, L.L.; Skarupski, K.A.; Scherr, P.A.; Evans, D.A. Neighborhood Social Cohesion and Disorder in Relation to Walking in Community-Dwelling Older Adults: A Multilevel Analysis. J. Aging Health 2009, 21, 155-171.

20. Lee, I.; Ewing, R.; Sesso, H.D. The built environment and physical activity levels: The Harvard Alumni Health Study. Am. J. Prev. Med. 2009, 37, 293-298.

21. Clark, A.F.; Scott, D.M. Does the social environment influence active travel? An investigation of walking in Hamilton, Canada. J. Transp. Geogr. 2013, 31, 278-285.

22. Chaudhury, H.; Mahmood, A.; Michael, Y.L.; Campo, M.; Hay, K. The influence of neighborhood residential density, physical and social environments on older adults' physical activity: An exploratory study in two metropolitan areas. J. Aging Stud. 2012, 26, 35-43.

23. Siu, V.W.; Lambert, W.E.; Fu, R.; Hillier, T.A.; Bosworth, M.; Michael, Y.L. Built Environment and Its Influences on Walking among Older Women: Use of Standardized Geographic Units to Define Urban Forms. J. Environ. Public Health 2012, 2012, 1-9.

24. Rodríguez, D.A.; Evenson, K.R.; Diez Roux, A.V.; Brines, S.J. Land use, residential density, and walking: The multi-ethnic study of atherosclerosis. Am. J. Prev. Med. 2009, 37, 397-404.

25. Shigematsu, R.; Sallis, J.; Conway, T.; Saelens, B.; Frank, L.; Cain, K.; Chapman, J.; King, A. Age differences in the relation of perceived neighborhood environment to walking. Med. Sci. Sport. Exer. 2009, 41, 314-321.

26. Zhang, Y.; Li, Y.; Yang, X.; Liu, Q.; Li, C. Built Environment and Household Electric Bike Ownership. Transport. Res. Rec. 2013, 2387, 102-111.

27. King, W.C.; Belle, S.H.; Brach, J.S.; Simkin-Silverman, L.R.; Soska, T.; Kriska, A.M. Objective measures of neighborhood environment and physical activity in older women. Am. J. Prev. Med. 2005, 28, 461-469.

28. Riva, M.; Gauvin, L.; Apparicio, P.; Brodeur, J.-M. Disentangling the relative influence of built and socioeconomic environments on walking: The contribution of areas homogenous along exposures of interest. Soc. Sci. Med. 2009, 69, 1296-1305.

29. Zhang, Y.; Li, Y.; Liu, Q.; Li, C. The Built Environment and Walking Activity of the Elderly: An Empirical Analysis in the Zhongshan Metropolitan Area, China. Sustainability 2014, 6, 1076-1092.

30. Badland, H.M.; Garrett, N.; Schofield, G.M. How does car parking availability and public transport accessibility influence work-related travel behaviors? Sustainability 2010, 2, 576-590.

31. Frank, L.; Kerr, J.; Chapman, J.; Sallis, J. Urban form relationships with walk trip frequency and distance among youth. Am. J. Health Promot. 2007, 21, 305-311. 
32. Cerin, E.; Lee, K.-Y.; Barnett, A.; Sit, C.; Cheung, M.-C.; Chan, W.-M.; Johnston, J. Walking for transportation in Hong Kong Chinese urban elders: A cross-sectional study on what destinations matter and when. Int. J. Behav. Nutr. Phys. Activity 2013, 10, 78-87.

33. Ewing, R.; Cervero, R. Travel and the Built Environment. J Am. Plann. Assoc. 2010, 76, 265-294.

34. Zhang, Y.; Chen, L.; Zhu, W.; Liu, X.; Chen, D. Relationship between physical activity and environment in Shanghai, China: Analysis and evaluation in adults aged 45-80. Med. Sport 2011, 64, 269-284.

35. National Bureau of Statistics of China. China Statistical Yearbook 2010; China Statistics Press: Beijing, China, 2010. (In Chinese)

36. Zhongshan Municipal Bureau of Urban Planning. Zhongshan Transportation Development Planning; Zhongshan Municipal Bureau of Urban Planning: Zhongshan, China, 2010. (In Chinese)

37. Martinez, L.M.; Viegas, J.M.; Silva, E.A. A traffic analysis zone definition: A new methodology and algorithm. Transportation 2009, 36, 581-599.

38. Ministry of Construction of the People's Republic of China. Code for Transport Planning on Urban Road (GB 50220-95); Ministry of Construction of the People's Republic of China: Beijing, China, 1995. (In Chinese)

39. Kockelman, K.M. Travel behavior as function of accessibility, land use mixing, and land use balance: Evidence from San Francisco Bay Area. Transport. Res. Rec. 1997, 1607, 116-125.

40. Ministry of Housing and Urban-Rural Development of the People's Republic of China. Code for Urban Land Use Classes and Standards of Planning Construction Land (GB50137-2011); Ministry of Housing and Urban-Rural Development of the People's Republic of China: Beijing, China, 2011. (In Chinese)

41. Zhao, P. The Impact of the Built Environment on Bicycle Commuting: Evidence from Beijing. Urban Stud. 2014, 51, 1019-1037.

42. Dill, J.; Voros, K. Factors affecting bicycling demand-Initial survey findings from the Portland, Oregon, Region. Transport. Res. Rec. 2007, 2031, 9-17.

43. Ball, K.; Jeffery, R.W.; Abbott, G.; McNaughton, S.A.; Crawford, D. Is healthy behavior contagious: Associations of social norms with physical activity and healthy eating. Int. J. Behav. Nutr. Phys. Activity 2010, 7, 86-95.

44. Yang, Z. Behavior and health. Chin. J. Behav. Med. Brain Sci. 2009, 18, 61-63. (In Chinese)

(C) 2014 by the authors; licensee MDPI, Basel, Switzerland. This article is an open access article distributed under the terms and conditions of the Creative Commons Attribution license (http://creativecommons.org/licenses/by/3.0/). 